\title{
Intelligent framework for diagnosis of frozen shoulder using cross sectional survey and case studies
}

\author{
Humaira Batool ${ }^{1}$, M. Usman Akram ${ }^{1 *}$, Fouzia Batool ${ }^{2}$ and Wasi Haider Butt ${ }^{1}$
}

*Correspondence:
usmakram@gmail.com
${ }^{1}$ National University
of Sciences and Technology,
Islamabad, Pakistan
Full list of author information
is available at the end of the
article

${ }^{*}$ Correspondence: usmakram@gmail.com of Sciences and Technology, Islamabad, Pakistan article

\begin{abstract}
Objectives: Frozen shoulder is a disease in which shoulder becomes stiff. Accurate diagnosis of frozen shoulder is helpful in providing economical and effective treatment for patients. This research provides the classification of unstructured data using data mining techniques. Prediction results are validated by K-fold cross-validation method. It also provides accurate diagnosis of frozen shoulder using Naïve Bayesian and Random Forest models. At the end results are presented by performance measure techniques.

Methods: In this research, 145 respondents (patients) with a severe finding of frozen shoulder are included. They are selected on premise of (clinical) assessment confirmed after by MRI. This data is taken from the department of Orthopedics (Pakistan Institute of Medical Sciences Islamabad and Railway Hospital Rawalpindi) between September 2014 to November 2015. Frozen shoulder is categorized on the basis of MRI result. The predictor variables are taken from patient survey and patient reports, which consisted of $35+$ variables. The outcome variable is coded into numeric system of "intact" and "no-intact". The outcome variable is assigned into numeric code, 1 for "intact" and 0 for "no-intact". "Intact" group is used as an indication that tissue is damaged badly and "nointact" is classified as normal. Distribution of result is 110 patients for "Intact" group and 35 patients for "No-Intact" group (false positive rate was $24 \%$ ). In this research we have utilized two methods i.e. Naive Bayes and Random Forest. A statistics regression model (Logistic regression) to categorize frozen shoulder finding into "intact" and "no-intact" classes. In the end, we validated our results by Bayesian theorem. This gives a rough estimate about the probability of frozen shoulder.
\end{abstract}

Results: In this research, our anticipated and predictive procedures gave better outcome as compared to statistical techniques. The specificity and sensitivity ratio of predicting a frozen shoulder are better in the Naiive Bayes as compared to Random Forest. In end the likelihood ratio results are used with Bayesian theorem for final evaluation of the results, from this we conclude predictive model is valid model for classification of frozen shoulder.

Conclusions: We have used three predictive models in our study to classify frozen shoulder. Then we validated our predictive results by Bayesian theorem to give a rough estimate about the probability of occurrence of disease or not. This enhances the clinical decision making regarding frozen shoulder. 


\section{Background}

The tendons and bones that make up our shoulder joint are enclosed in a capsule of connective tissue. Whenever shoulder capsule become tightened and thick around the joint, the problem of frozen shoulder occurs (Crubbs 1993). Mostly frozen shoulder problem can be categorized into either primary also called unknown reason (idiopathic) or secondary (known reason) (Dias et al. 2005). Main signs and symptoms which lead to frozen shoulder are bad posture, post-operative (after surgery), stroke and patients suffering from diabetes (Mao et al. 1997). Frozen shoulder is more prone diabetics $(20 \%)$ than general population (2-5\%).Its ratio in insulin dependent patients are about $36 \%$ (Afsar et al. 2014). Females are affected more than males (Manske and Prohaska 2010). Many treatments are available for frozen shoulder including both operative and non-operative. Operative procedures include manipulation under anesthesia and arthroscopic surgeries. Surgery reduces the severe complications of frozen shoulder. Non-operative includes pain management through different modalities (Transcutaneous electrical nerve stimulation, Short wave diathermy, Interferential therapy etc.), mobilization techniques, exercise plan and precautionary measures. All treatments just improve the functional time to recovery and increase the range of motion (Neviaser 1945). However, none of the treatment is authentic to totally eliminate the future chance of disease.

Main symptoms observed in frozen shoulder patients are pain, stiffness and loss of range of motion (Crubbs 1993). According to our knowledge, no work has been done on frozen shoulder using unstructured data (Ahmad et al. 2012). Only a few studies have been performed on clinical examinations and treatments with medical perspective, but no work has been done for the diagnosis of a frozen shoulder with respect to predictor values. The objective of this research is to develop reliable method for diagnosing frozen shoulder. Another significance of this study, is to analyze frozen shoulder intelligently by using unstructured data.

Physical examinations are generally considered low-cost process and results can directly be obtained at the time of the consultation. On the other hand, precision is based upon doctor's knowledge and practice. Now a day's frozen shoulder is diagnosed by clinical examination and imaging tests (Değerlendirmesi 2014). Firstly doctor diagnoses the problem by asking the patient to rotate the shoulder in a different direction. If physicians are uncertain about the problem, then formal test such as magnetic resonance imaging (MRI) can be carried out for diagnosis. Arthography is considered a standard test for the diagnosis of a frozen shoulder. It is having sensitivity $91 \%$, specificity $100 \%$ and accuracy $92 \%$, but the test is an expensive and painful process (Ryu et al. 1993).

Better diagnosis and treatment plan for frozen shoulder are made through clinical findings. A lot of clinical examination methods have been developed to help in diagnosing frozen shoulder (Manske and Prohaska 2008). If patient is having limitations in hand elevation, then Apley scratch test is used for the diagnosis of disease. In Apley scratch test, patient is asked to put his/her arm above head and arrive at behind the neckline to touch his/her upper back. This test analyzes the rotation of upward, external and elevation (Anderson et al. 2011). During physical examination if the patient is having severe pain, then the physical assessment is marked positive. Previous researches showed that the ROM (range of motion) and Apley scratch test are good for diagnosing frozen shoulder (Woodward and Best 2000). 
There is no existing fact, that any solitary check can diagnose frozen problem (Mitchell et al. 2005). Cost-effective treatment process is always dependent upon proper clinical assessment and diagnosis. The severity of the disease can be judged best by imaging tests as compared to clinical examination. According to the recent researches, if we make decision just on the basis of physical examination, it has sure chances that to give us false-positive ratio. Analysis made on the basis of physical examination always conflict with the imaging test. Due to this, we cannot make any decision just on the basis of clinical examination (Manske and Prohaska 2008; Bulgen et al. 1984; Clarke et al. 1974; Binder et al. 1984; Shaffer et al. 1992; Sharma et al. 1993).

This research provides a pathway towards accurate and correct diagnosis of frozen shoulder. The objective of this research is to develop reliable method for frozen shoulder. First milestone is to collect questionnaire from work related and chronic disease patients. Then on the basis of questionnaire and physical examination reports, we develop model which detect frozen shoulder category.

Identification of frozen problem by intelligence based framework is a new research area because previously it was based upon clinical examination. Clinical examination accuracy is dependent upon physician experience. Few case studies based researches have been done on clinical examination for the diagnosis of different shoulder problems but no research has been done on the correct diagnosis of shoulder problem by using intelligence framework. It may cause life time abnormality if not monitored well on time. So intelligence test should be used as a side assessment, for correct diagnosis of shoulder problem.

Data mining process is called as computational process. During computational process, software matches different patterns on the basis of logics. It classifies the huge datasets using different techniques like statistics and machine learning [ML] (Han et al. 2011; Bellazzi and Zupan 2008). This information discovery procedure turned out to be an accepted area to make inquires in diverse fields. It is being used in medical domain to discover patterns among medical variables. This also predicts disease outcomes using historical data (Bellazzi and Zupan 2008; Ramesh et al. 2004).

It is a difficult task for a doctor to properly diagnose and recommend treatment for some serious disease problems. In this context intelligent system model is considered as a useful procedure. We took information from different patients and then forecast a conclusion of interest (Bellazzi and Zupan 2008; Witten and Frank 2005). This will help us to take decision at a clinical level. Predictive methods of data mining are, Naïve Bayesian and Random Forest. These have been used most of the time to predict the conclusion of disease (Bellazzi and Zupan 2008; Griffith 2000). Some relevant studies used this concept; to provide prediction about how many patients are at high risk during anesthesia (Lin et al. 2011). Another study was on internal shoulder derangements (Oh et al. 1999).

Our main objective is to build up a procedure to diagnose frozen shoulder and to make decisions on the basis of physical information without depending on tests like MRI. Tests should only be used for detailed clinical hypothesis and only be recommended in severe and undiagnosed cases (Weinstein et al. 1980; Kassirer 1989; Pauker and Kassirer 1980). This study also used a predictive procedure of data mining and Chi Square test, to increase the precision of diagnosing frozen shoulder without physical examination. In this study we build a model and also made comparison among three predictive 
models (logistic regression, Random forest and Naïve Bayes) to categorize frozen shoulder groups on the basis of physical examination results, patient reports and survey.

\section{Proposed methodology}

This research classifies the unstructured data using data mining techniques. It also used predictive models like Naïve Bayesian and Random forest to improve the diagnosis of frozen shoulder.

Methodology comprises of feasibility study, data gathering, preprocessing, and detecting predictor attributes. Then on the basis of training data, result was graded into different meaningful classification.

\section{Architecture}

This research used three tier architecture levels for analysis of disease. Figure 1 shows the architecture.

\section{Requirement level}

This consists of collecting and finalizing data at single point. It also includes linkage of data with datasets level, to build an intelligent system for problem analysis. Requirement engineering $[R E]$ process suggests the most common requirements according to the needs.

\section{Datasets level}

This mainly deals with database. Database consists of datasets.

\section{Component level}

This consists of framework to classify data.

\section{Block diagram of proposed system}

Figure 2 shows the block diagram of the proposed system. The data we collected from patient reports and patient survey was in an unstructured form. Unstructured data mean raw data. Almost $80 \%$ of medical data are available in an unstructured form like patient's reports, lab report, and doctor review. We have collected history of different patients by case study method, for the purpose to get some predictor variables. All those

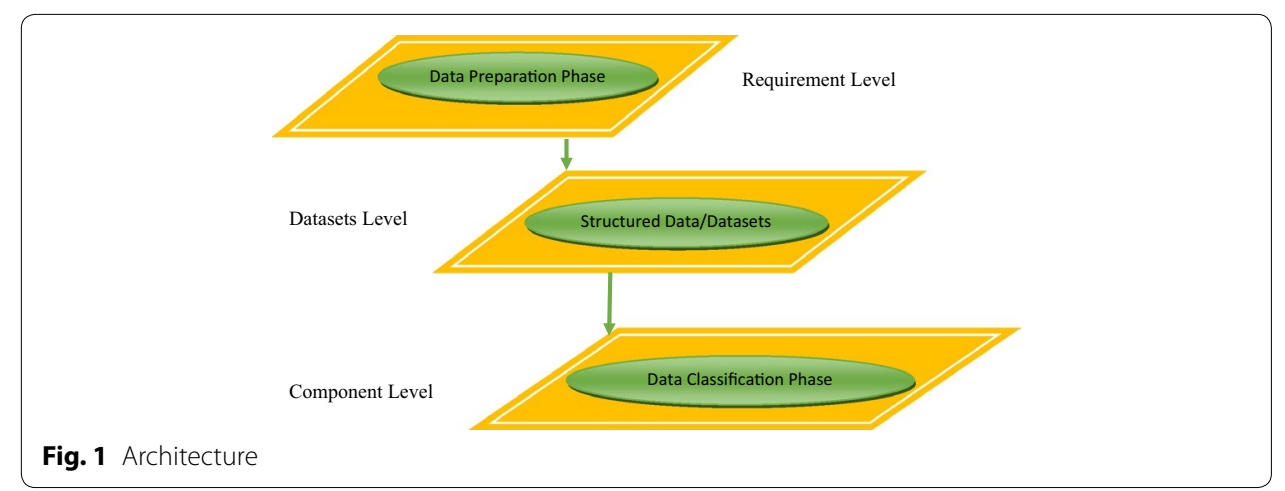




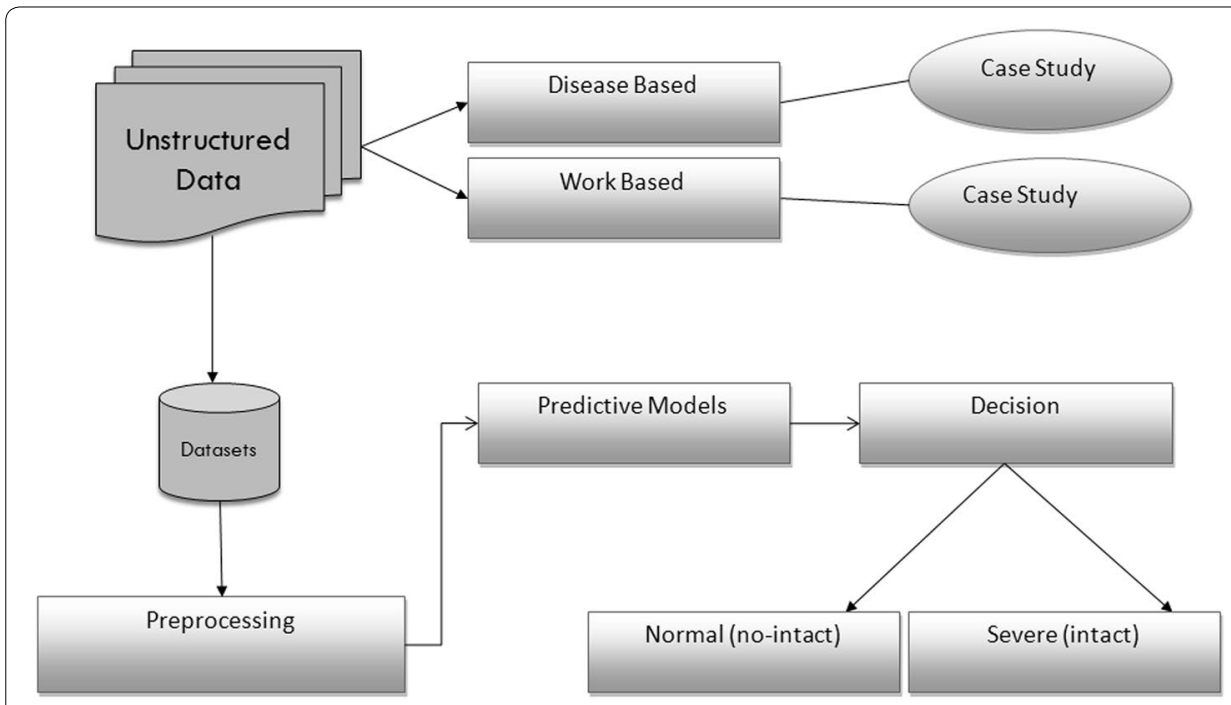

Fig. 2 Block diagram of proposed system

case studies reveal that either the frozen shoulder problem occurred due to work related problem or either it is due to disease base problem. In the second level of architecture we converted unstructured data of patients into structured form by using software. Structure data was analyzed by the software. Then preprocessing was done on a datasets it includes feature extraction. Details about feature extraction method are mentioned in below section. In the third level of architecture we have applied different predictive model to classify unstructured data. This will also help in deciding either frozen shoulder patient classifies into intact class or no-intact class.

\section{Requirement engineering process}

In our research study, we have used a well known requirement engineering process model for data preparation. Requirement engineering process is called as "The process to gather the requirements from client analyze and document them". Data preparation output would be used for predicting result accurately and intelligently. Client involvement may be a major consideration in the development of most successful systems. The main purpose of using requirement engineering process in our study is that, the most difficult phase is to collect patient's information and user involvement for finalizing all data at some single point. So far it is largely unexplored. Model defines the approaches and methods used to deal with difficulties.

Requirement engineering is a four step process, which includes feasibility study, requirement gathering, requirement specification, and requirement validation. Each stage has its specific tasks and outputs.

The emphasis of first step is on getting better understanding of project objectives to establish better plans to achieve business and intelligent goals. Feasibility study is an analysis of the importance of an idea. Conducting a feasibility study is a good practice to gives focuses to the project and outline alternatives. Next step includes data collection from the patient and doctors. It involves communicate with the patient and doctors to know their views about disease and to identify important features for survey. Data understanding is 
achieved by categorizing data into work based, disease based. After finalizing the requirements next phase of the process is requirement specification, requirement specification is same like blueprints. Framework selection according to domain is an art which can easily tie all internal factors of requirements. Framework design usually utilize the most time of the project. When business goals are settled then next step is requirement validation. Requirement validation step involves to check whether all important points are covered correctly and easily validate through alternatives. It also confirms that whether all domain requirements are accurately adjusted in a document or not.

This study was conducted with the collaboration of Pakistan Institute of Medical Science Islamabad and Railway Hospital Rawalpindi, Pakistan. A real report based data of frozen shoulder patients was used. In this study, original data set is used that was obtained from Hospitals. For the purpose of domain understating we covered different case studies. This composed data was unstructured patient's records. First we transformed the unstructured reports/survey into planned order and then picked out important variables. By hands mean, we have selected some hidden predictive variables from the patient reports, which were obtained from the information doctor has taken during the subjective examination or history taking. We designed a patient survey with the assistance of therapeutic specialists (practitioners).Extraction of meaningful information from the survey was done very carefully. The requirement engineering process was used to decide whether the extracted attributes are sufficient and contains all the meaningful attributes or still extra information is required to get important variables. The mining (extraction) of variables is key benefit to get the knowledge of problem field plus it would assist additionally within the study. After the categorization of data, datasets was formed. Requirement analysis steps are shown in a Fig. 3.

\section{Significance of irregular (unstructured) data}

In 1998, Merrill Lynch declared that a normal $80 \%$ of all business information has it source in unstructured form (Murdoch and Detsky 2013). Mostly larger part of business information should came from unstructured source. As indicated by another source

\section{Requirement Analysis Phase}

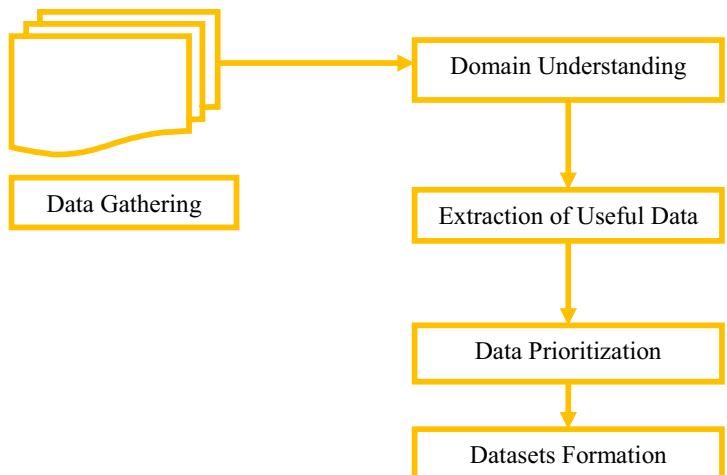

Fig. 3 Requirement analysis phase 
(Murdoch and Detsky 2013), around $80 \%$ of the medical information that might be patient's reports is in irregular form. Once the unorganized information is organized and put in database is called planned data. The planned information is easily comprehensible by computers and is used by framework for problem analysis.

\section{Datasets level}

We have collected meaningful information from last level which would be helpful for decision. We designed the datasets to grasp the collected variables. The patient survey data set contained different variables which were categorical, nominal and scale data. The description and values of patient's survey variables are stated in Table 1 . The description and values of patient's reports variables are stated in Table 2.

\section{Data collection procedure}

In this research, 145 respondents (patients) with severe symptoms of frozen shoulder were included. They were selected on the premise of (clinical) assessment confirmed after by MRI. This data was taken from the department of Orthopedics (Pakistan Institute of Medical Sciences Islamabad and Railway Hospital Rawalpindi) between September 2014 to November 2015. Frozen shoulder was categorize on the basis of MRI result. MRI result was used a standard.

The result variable was coded into numeric system of "intact" and "no-intact". "Intact" group incorporated that tissue has been damaged badly and "no-intact" was classified as normal tissue. The result distribution variable was 110 for "Intact" and 35 patients for "No-Intact" (False positive rate of $24 \%$ ). The judgment variable was the clinical results, which consist of 35+ variables. Apley's Scratch Test and Resisted Tests (ROM) are common provocative tests in diagnosing frozen shoulder (Woodward and Best 2000). These two tests were utilized as screening/diagnostic tests in the analysis models. In this research, we have utilized three mining methods to categorize frozen shoulder (intact and no-intact classes).it includes, Naive bayes and the Random Forest and a statistics regression model (logistic regression). Dataset consisted of surveyed questions and reports. It contained all the data on the subject of abnormalities and their relation, as it is used for assessment and association purposes. However, for this study there was no availability of dataset either locally or globally. Due to this, we have collected data from different hospitals by survey method. The assessment of proposed framework of frozen shoulder patients was performed on a locally gathered dataset.

\section{Methods}

Precision in medical systems is extremely significant. We have used three tier architecture. It includes requirement level, datasets level and component level. First two levels have already been explained in the previous section. Component level includes feature extraction and classification techniques. The study also helps in comparison with different classification techniques.

This paper includes proposed framework results and details of dataset. Comparison with the other techniques is also included for the validation of proposed system. We used statistics regression model (logistic regression) to assess the outcomes (Lin et al. 2010; Bagley et al. 2001). The result of this model is then compared with other two 
Table 1 Variable list of patient survey

\begin{tabular}{|c|c|c|}
\hline Variables & Type & Coding \\
\hline \multicolumn{3}{|l|}{ Outcome } \\
\hline Frozen shoulder & Nominal & 2 codes ( 1 for Yes, 0 for No) \\
\hline \multicolumn{3}{|l|}{ Predictor } \\
\hline Age & Ordinal & 3 codes $(20-45,46-60, \geq 61)$ \\
\hline Gender & Nominal & 2 codes ( 1 for male, 0 for female) \\
\hline Occupation & Nominal & 4 codes \\
\hline Hand & Nominal & 2 codes ( 1 for right, 2 for left) \\
\hline BMI & Ordinal & 3 codes \\
\hline Conditions & Nominal & 14 codes \\
\hline Medications & Nominal & 11 codes \\
\hline Smoke & Nominal & 2 codes ( 1 for yes, 0 for no) \\
\hline Stress & Ordinal & 3 codes \\
\hline Problem & Nominal & 6 codes \\
\hline Complaint & Nominal & 6 codes \\
\hline Shoulder & Nominal & 2 codes ( 1 for right, 2 for left) \\
\hline Sharp pain & Nominal & 2 codess ( 1 for yes, 0 for no) \\
\hline Dull Pain & Nominal & 2 codes ( 1 for yes, 0 for no) \\
\hline Aching & Nominal & 2 codes ( 1 for yes, 0 for no) \\
\hline Throbbing & Nominal & 2 codes ( 1 for yes, 0 for no) \\
\hline Numbness & Nominal & 2 codes ( 1 for yes, 0 for no) \\
\hline Shooting & Nominal & 2 codes ( 1 for yes, 0 for no) \\
\hline Burning & Nominal & 2 codes ( 1 for yes, 0 for no) \\
\hline Pain & Nominal & 4 codes \\
\hline Pain score & Nominal & 2 codes \\
\hline Wake & Nominal & 2 codes ( 1 for yes, 0 for no) \\
\hline Before & Nominal & 2 codes ( 1 for yes, 0 for no) \\
\hline Dislocated & Nominal & 2 codes ( 1 for yes, 0 for no) \\
\hline Injection & Nominal & 2 codes ( 1 for yes, 0 for no) \\
\hline Surgery & Nominal & 2 codes ( 1 for yes, 0 for no) \\
\hline Medicine & Nominal & 2 codes ( 1 for yes, 0 for no) \\
\hline Interfere & Nominal & 2 codes ( 1 for yes, 0 for no) \\
\hline Activity & Nominal & 2 codes ( 1 for yes, 0 for no) \\
\hline Lifting & Nominal & 2 codes ( 1 for yes, 0 for no) \\
\hline Behind & Nominal & 2 codes ( 1 for yes, 0 for no) \\
\hline Sports & Nominal & 2 codes ( 1 for yes, 0 for no) \\
\hline Decrease & Nominal & 4 codes \\
\hline MRI & Nominal & 2 codes ( 1 for yes, 0 for no) \\
\hline Repetitive & Nominal & 2 codes ( 1 for yes, 0 for no) \\
\hline Hours & Nominal & 5 codes \\
\hline Improvement & Nominal & 3 codes \\
\hline Killers & Nominal & 2 codes ( 1 for yes, 0 for No) \\
\hline Trauma & Nominal & 2 codes ( 1 for yes, 0 for No) \\
\hline Working & Nominal & 2 codes ( 1 for yes, 0 for No) \\
\hline Dominant & Nominal & 5 codes \\
\hline History & Nominal & 10 codes \\
\hline
\end{tabular}

Classification Techniques (which are Nave Bayes and Random Forest). We have used cross validation techniques for performance authentication. Verification was done by comparing the accuracy of classification models. 
Table 2 Variable list of patient report

\begin{tabular}{lll}
\hline Variables & Type & Coding \\
\hline Active range of motion & Nominal & 2 codes ( 1 for positive, 0 for negative $)$ \\
Passive range of motion & Nominal & 2 codes ( 1 for positive, 0 for negative) \\
Internal rotation & Nominal & 2 codes ( 1 for positive, 0 for negative $)$ \\
External rotation & Nominal & 2 codes ( 1 for positive, 0 for negative $)$ \\
Flexion & Nominal & 2 codes ( 1 for positive, 0 for negative $)$ \\
Abduction & Nominal & 2 codes ( 1 for positive, 0 for negative $)$ \\
Apley's scratch test & Nominal & 2 codes ( 1 for positive, 0 for negative $)$ \\
\hline
\end{tabular}

\section{Feature selection}

In this step unrelated variables and unnecessary information was eliminated. At the start numbers of variables were $35+$, extracted from the survey and reports. A few of the variables were unnecessary and was not helpful in decision making. Those variables were removed by feature selction method. Data preprocessing includes feature selection, which helps in decreasing the unrelated data and improves the learning effectiveness. This also increases the accuracy of models. After this procedure, resulted variables are those variables that are helpful for identification of purpose and also produce high accuracy for classification models. Feature extraction was done by $T$ TEST technique and significance probability value was taken as 0.05 .

Most of the research questions have been validated through hypothesis. The research questions are for our knowledge and therefore the statistics that comes hereafter. Whenever, we have created hypothesis test in statistics, a P value always help us to conclude the importance of our outcome. Basically hypothesis tests are used to check the strength of a claim that is made about a community. This claim is known as the null hypothesis. After the outcome, if the null hypothesis is inaccurate then we would accept the other hypothesis as true. In statistics, $\mathrm{P}$ value is a range among Zero and One and translated in the subsequent way:

- After study if results show small P value $(\leq 0.05)$, then we can easily reject the null hypothesis because smallest $P$ value shows strong evidence against the null hypothesis.

- After study if results show large P value (>0.05), then we cannot reject the null hypothesis because large P value shows weak evidence against the null hypothesis.

- After study if results show P values near the cutoff (0.05), then we could go either way because results are on margin.

\section{Component level (classification phase)}

In this section, we would explain in detail about the component steps. It is used to categorize the data. We will compare different classification techniques. At the end, conclusion can be made on the basis of comparison results.

\section{Training and testing procedure}

Generally the error is related with randomness results in biasing the estimation. Cross-validation is regularly applied to minimize the error. The power of a prediction model to generalize unseen data can be frequently validated by K-fold cross validation method. All sampling data is divided into K equal size subsamples randomly, by K-fold 
cross-validation procedure strategy. We have used $50 \%$ data for testing purpose and $50 \%$ data for training purpose. The both method (training and testing) is then repeated $\mathrm{K}$ time. In this study, K-fold cross-validation number ten was chosen in light of the fact that numerous researches have demonstrated that ten as an ideal validation number. In the tenfold cross-approval, the method was repeated ten times with totally dissimilar training and testing datasets (Kohavi 1995; Bengio and Grandvalet 2004; Breiman et al. 1984).

We have additionally checked the error of different predictive models by performance measures method during comparative analysis (Delen et al. 2005).

\section{Results}

We have collected data from 145 frozen shoulder patients by survey and patient's real reports. Table 3 shows the demographic data of the patients. The majority patients were Female (71\%); the age ranges were in between $46-60$ years $(44.8 \%)$.

Majority respondents were employee (44.8\%) and housewife. Majority of the patients dominant hand was right (86.9 \%) and their body mass was normal (55.2 \%). Majority of the patients were suffering from HBP-diabetes-depression (37.9 \%).Stress level in majority of patients was high due to occupation (57.9\%). Left shoulder was involved in majority of the respondent $(60.7 \%)$.Those patients wake at night with pain ratio was (86.2\%); (53.8 \%) respondents were having main complaint of the pain-stiffness. History of complain; due to work related activities were present in (43.4\%) patients. Origin of pain was sudden in (39.3\%) respondents and nearly $58.6 \%$ of patients rated their pain as worst. The ratio of patients with no such complaint before was (72.4\%). Majority was not having any previous history of dislocation, injection in a shoulder and before history of surgery and (80 \%) patients were on medication. Majority respondents told that pain interferes with their routine work (96.6\%) and also with their sports activities. (55.9\%) patients told that after heat therapy they feel better but that is just for some time. Majority said that repetitive lifting loads at their work place are main cause of pain and almost (77.2 \%) were using pain killers for musculoskeletal problems (77.2 \%).

Clinical examination is being done mostly on the basis of Active range of motion, internal rotation, flexion and Apley's scratch. These are considered as standard interpreter attributes to decide the frozen shoulder. The proportions of positive finding were 97.2, 89.0, 93.1 and $89.0 \%$ respectively. Respondent, were having negative passive range of motion, external rotation were positive $91.7 \%$, Abduction test were positive 42.8 as shown in Table 4. The assessment attributes also included different kind of pain types (which are sharp, aching, throbbing, numbness, shooting, burning pain) which were then coded into two responses. The response having sharp pain was $(82.1 \%)$ and percentage of aching pain was (64.8\%).The throbbing pain was (95.9\%). Some patients had numbness $(80 \%)$, burning $(77.2 \%)$ and shooting ratio was $91.0 \%$.

The P value was used to look at the valuable attribute between the "intact" and "nointact" class. Table 5 shows the valuable attribute between Intact and No-Intact class. The asterisk (*) code in a last column shows the significance of each predictor variable. Variables with ${ }^{* * * *}$ code are the most significant ones and all others variable without any * has no significance association. Along with MRI report, we have assigned class labels to every record. 
Table 3 Demographic Data of the 145 Patients

\begin{tabular}{|c|c|c|c|}
\hline Description & Characteristics & Frequency & Percentage \\
\hline \multirow[t]{3}{*}{ Age } & $20-45$ & 48 & 33.1 \\
\hline & $46-60$ & 65 & 44.8 \\
\hline & $\geq 61$ & 32 & 22.1 \\
\hline \multirow[t]{2}{*}{ Gender } & Male & 42 & 29.0 \\
\hline & Female & 103 & 71.0 \\
\hline \multirow[t]{4}{*}{ Occupation } & Housewife & 63 & 43.4 \\
\hline & Employee & 65 & 44.8 \\
\hline & Un-employee & 02 & 1.4 \\
\hline & Retired & 15 & 10.3 \\
\hline \multirow[t]{2}{*}{ Hand } & Right & 126 & 86.9 \\
\hline & Left & 19 & 13.1 \\
\hline \multirow[t]{3}{*}{ BMI } & Overweight & 17 & 11.7 \\
\hline & Underweight & 48 & 33.1 \\
\hline & Normal weight & 80 & 55.2 \\
\hline \multirow[t]{9}{*}{ Conditions } & Arthritis & 01 & 0.7 \\
\hline & Diabetes & 07 & 4.8 \\
\hline & Depression & 01 & 0.7 \\
\hline & Other & 02 & 1.4 \\
\hline & Diabetes-depression & 23 & 15.9 \\
\hline & HBP-diabetes-depression & 55 & 37.9 \\
\hline & Difficulty sleeping-HBP-diabetes-depression & 52 & 35.9 \\
\hline & Arthritis-HBP & 02 & 1.4 \\
\hline & HBP-diabetes-depression-heart Disease & 02 & 1.4 \\
\hline \multirow[t]{2}{*}{ Smoke } & Yes & 27 & 18.6 \\
\hline & No & 118 & 81.4 \\
\hline \multirow[t]{3}{*}{ Stress } & High & 84 & 57.9 \\
\hline & Medium & 54 & 37.2 \\
\hline & Low & 07 & 4.8 \\
\hline \multirow[t]{2}{*}{ Shoulder } & Right & 57 & 39.3 \\
\hline & Left & 88 & 60.7 \\
\hline \multirow[t]{2}{*}{ Wake } & Yes & 125 & 86.2 \\
\hline & No & 20 & 13.8 \\
\hline \multirow[t]{5}{*}{ Problem } & Pain & 30 & 20.7 \\
\hline & Stiffness & 10 & 6.9 \\
\hline & Dislocation & 02 & 1.4 \\
\hline & Pain-stiffness & 78 & 53.8 \\
\hline & Pain-stiffness-dislocation & 25 & 17.2 \\
\hline \multirow[t]{6}{*}{ Complain } & Injury & 16 & 11.0 \\
\hline & Work-based & 63 & 43.4 \\
\hline & Disease-based & 42 & 29.0 \\
\hline & Surgery & 01 & 0.7 \\
\hline & Injury-work-based & 05 & 3.4 \\
\hline & Injury-disease-based & 18 & 12.4 \\
\hline \multirow[t]{4}{*}{ Pain } & Constant & 33 & 22.8 \\
\hline & Frequent & 52 & 35.9 \\
\hline & Occasional & 57 & 39.3 \\
\hline & Intermittent & 03 & 2.1 \\
\hline \multirow[t]{2}{*}{ Pain Score } & Average & 60 & 41.4 \\
\hline & Worst & 85 & 58.6 \\
\hline
\end{tabular}


Table 3 continued

\begin{tabular}{|c|c|c|c|}
\hline Description & Characteristics & Frequency & Percentage \\
\hline \multirow[t]{2}{*}{ Before } & Yes & 40 & 27.6 \\
\hline & No & 105 & 72.4 \\
\hline \multirow[t]{2}{*}{ Dislocated } & Yes & 38 & 26.2 \\
\hline & No & 107 & 73.8 \\
\hline \multirow[t]{2}{*}{ Injection } & Yes & 52 & 35.9 \\
\hline & No & 93 & 64.1 \\
\hline \multirow[t]{2}{*}{ Surgery } & Yes & 30 & 20.7 \\
\hline & No & 115 & 79.3 \\
\hline \multirow[t]{2}{*}{ Medicine } & Yes & 116 & 80 \\
\hline & No & 29 & 20 \\
\hline \multirow[t]{2}{*}{ Interfere } & Yes & 140 & 96.6 \\
\hline & No & 05 & 3.4 \\
\hline \multirow[t]{2}{*}{ Activity } & Yes & 121 & 83.4 \\
\hline & No & 24 & 16.6 \\
\hline \multirow[t]{2}{*}{ Lifting } & Yes & 139 & 95.9 \\
\hline & No & 06 & 4.1 \\
\hline \multirow[t]{2}{*}{ Behind } & Yes & 140 & 96.6 \\
\hline & No & 05 & 3.4 \\
\hline \multirow[t]{2}{*}{ Sports } & Yes & 30 & 20.7 \\
\hline & No & 115 & 79.3 \\
\hline \multirow[t]{4}{*}{ Decrease } & Heat & 81 & 55.9 \\
\hline & Ice & 01 & 0.7 \\
\hline & Rest & 09 & 6.2 \\
\hline & Rest-heat & 54 & 37.2 \\
\hline \multirow[t]{2}{*}{ MRI } & Yes & 110 & 75.9 \\
\hline & No & 35 & 24.1 \\
\hline \multirow[t]{2}{*}{ Repetitive } & Yes & 112 & 77.2 \\
\hline & No & 33 & 22.8 \\
\hline \multirow[t]{2}{*}{ Killers } & Yes & 112 & 77.2 \\
\hline & No & 33 & 22.8 \\
\hline \multirow[t]{2}{*}{ Trauma } & Yes & 18 & 12.4 \\
\hline & No & 127 & 87.6 \\
\hline
\end{tabular}

Probability method evaluated the significance of an attribute by computing the worth of $\mathrm{P}$ value measurement. It uses ranker search technique with threshold $\mathrm{P} \leq 0.05$. This search method gives weight and $\mathrm{P}$ value by their individual evaluations. Mostly $\mathrm{P}$ value was used to check the similarity between the Intact and No-Intact groups of each variable. Those variables which have mentioned in Table 5 with Starric $(* *)$ codes, are selected due to their $P$ value less than 0.05 . Four $\left({ }^{* * * *}\right)$ code variables are more significant follow by three codes, two codes and one code. One $\left(^{*}\right)$ code is least significant due to having more $\mathrm{P}$ value among the other significant variables. The performance of the proposed system is evaluated on significant variables. The effective attributes are shown in Table 5.

Firstly we separated variables against the code. According to the coding scheme, we have separately applied model on different codes and then judged the accuracy of different variable codes. We have also validated our results and calculated the accuracy by the formula given in Eq. 1. 
Table 4 Symptoms related data of patients

\begin{tabular}{|c|c|c|c|}
\hline Description & Characteristics & Frequency & Percentage \\
\hline \multirow[t]{2}{*}{ Sharp pain } & Yes & 119 & 82.1 \\
\hline & No & 26 & 17.9 \\
\hline \multirow[t]{2}{*}{ Aching } & Yes & 94 & 64.8 \\
\hline & No & 51 & 35.2 \\
\hline \multirow[t]{2}{*}{ Throbbing } & Yes & 139 & 95.9 \\
\hline & No & 06 & 04.1 \\
\hline \multirow[t]{2}{*}{ Numbness } & Yes & 116 & 80 \\
\hline & No & 29 & 20 \\
\hline \multirow[t]{2}{*}{ Shooting } & Yes & 13 & 9.0 \\
\hline & No & 132 & 91.0 \\
\hline \multirow[t]{2}{*}{ Burning } & Yes & 112 & 77.2 \\
\hline & No & 33 & 22.8 \\
\hline \multirow[t]{2}{*}{ Active range of motion } & Positive & 141 & 97.2 \\
\hline & Negative & 04 & 02.8 \\
\hline \multirow{2}{*}{ Passive range of motion } & Positive & 48 & 33.1 \\
\hline & Negative & 97 & 66.9 \\
\hline \multirow[t]{2}{*}{ Internal rotation } & Positive & 129 & 89.0 \\
\hline & Negative & 16 & 11.0 \\
\hline \multirow[t]{2}{*}{ External rotation } & Positive & 133 & 91.7 \\
\hline & Negative & 12 & 8.3 \\
\hline \multirow[t]{2}{*}{ Flexion } & Positive & 135 & 93.1 \\
\hline & Negative & 10 & 6.9 \\
\hline \multirow[t]{2}{*}{ Abduction } & Positive & 62 & 42.8 \\
\hline & Negative & 83 & 57.2 \\
\hline \multirow[t]{2}{*}{ Apley's scratch test } & Positive & 129 & 89.0 \\
\hline & Negative & 16 & 11.0 \\
\hline
\end{tabular}

(Valid number of predictions $* 1.00 /$ Total number of patients) $* 100$

\section{Models comparative analysis}

We have used different prediction models for this study. Firstly, we have analyzed the data by logistic regression model. After model implementation, we have used two other classification techniques for the purpose of analysis and comparison with Logistic Regression. The following results were obtained: LR model classified data with $96.55 \%$ accuracy during training of model. LR model gave $95.10 \%$ accuracy during cross validation. We have applied the same steps on Naïve Bayes and Random Forest. Table 6 results show that Random Forest categorizes the data $94.48 \%$ while training, it gives $89.81 \%$ accuracy during cross validation time. For Naïve Bayes we achieved $99.31 \%$ accuracy during training of model, it gives $99.29 \%$ accuracy during cross validation. Overall results show that, Naïve Bayes model performs most excellent on our data along with other procedures and techniques. Table 6 shows the classification models results.

Table 6 shows the prediction performances measure of AUC and etc. According to the above result, each metric depicts that Naïve Bayes performance are best than other models. Naïve Bayes is with highest accuracy. Along with Naïve Bayes, LR also shows immense performance that is $95.10 \%$. Naive Bayes has most favorable precision (100\%), sensitivity (97.5\%), specificity (100\%), accuracy (99.29), AUC(0.99) and likelihood 
Table 5 Valuable variables between intact and no-intact group

\begin{tabular}{|c|c|c|c|c|c|c|c|}
\hline S. no & Variable & P value & Code & S. no & Variable & $P$ value & Code \\
\hline 01 & Age & 0.670 & & 21 & Before & 0.342 & \\
\hline 02 & Gender & 0.001 & $* * * *$ & 22 & Dislocated & 0.434 & \\
\hline 03 & Occupation & 0.002 & $* * *$ & 23 & Injections & 0.089 & * \\
\hline 04 & Hand & 0.386 & & 24 & Surgery & 0.958 & \\
\hline 05 & $\mathrm{BMl}$ & 0.896 & & 25 & Medicines & 0.037 & $* *$ \\
\hline 06 & Conditions & 0.894 & & 26 & Interfere & 0.933 & \\
\hline 07 & Smoke & 0.590 & & 27 & Activity & 0.465 & \\
\hline 08 & Stress & 0.147 & & 28 & Lifting & 0.928 & \\
\hline 09 & Problem & 0.003 & $* * *$ & 29 & Behind & 0.933 & \\
\hline 10 & Complaint & 0.680 & & 30 & Sports & 0.390 & \\
\hline 11 & Shoulder & 0.991 & & 31 & Repetitive & 0.004 & $* * *$ \\
\hline 12 & Sharp & 0.297 & & 32 & Killers & 0.010 & $* *$ \\
\hline 13 & Ache & 0.787 & & 33 & Trauma & 0.530 & \\
\hline 14 & Throbbing & 0 & $* * * *$ & 34 & Active ROM & 0.435 & \\
\hline 15 & Numbness & 0.001 & $* * * *$ & 35 & Passive ROM & 0.486 & \\
\hline 16 & Shooting & 0 & $* * * *$ & 36 & Abduction & 0.446 & \\
\hline 17 & Burning & 0 & $* * * *$ & 37 & Internal & 0 & $* * * *$ \\
\hline 18 & Pain & 0.008 & $* * *$ & 38 & External & 0.000 & $* * * *$ \\
\hline 19 & Score & 0.171 & & 39 & Flexion & 0.000 & $* * * *$ \\
\hline 20 & Wake & 0.009 & $* * *$ & 40 & Apley & 0 & $* * * *$ \\
\hline
\end{tabular}

Table 6 Classification models results with prediction performance

\begin{tabular}{lllccl}
\hline Algorithm & Precision & AUC & Specificity & Sensitivity & Overall accuracy \\
\hline Logistic regression & 89.67 & 0.96 & $91.67(0.9167)$ & $96.7(0.967)$ & 95.1 \\
Naïve bayes & 100 & 0.99 & $97.5(0.975)$ & $100(1)$ & 99.29 \\
Random forest & 95 & 0.89 & $61.67(0.6167)$ & $99.09(0.9909)$ & 89.81 \\
\hline
\end{tabular}

positive and negative ratios (40,0).Logistic Regression results were also similar to recognize frozen shoulder with sensitivity (91.67), specificity (97.7), precision (89.67), accuracy (95.1), AUC (0.96) and likelihood positive and negative ratios $(11,0.04)$.The predictive data mining models Naïve Bayes, Logistic Regression has statistically better performances than the Random Forest.

In end we adopted the area under the receiver operating characteristics (AUROC). AUROC was used to analyze the biasness, which is basically used to differentiate those who have an Intact or who don't. Mostly the predictive models accuracy is explained by the area under the curve. We can classify the accuracy of predictive models by matching the points. If result shows value (0.90-1) then the diagnostic test is excellent, for good its value is $(0.80-0.90)$. Table 6 shows the predictive models along with their AUC value.

The areas under the curve for Naïve bayes is 0.99 and for logistic regression its 0.96 and for random forest its 0.89 . Overall values shows this Naïve Bayes would be considered to be excellent for separating intact from no-intact group.

The study conclude this Naïve Bayes performed more accurately in TPR (Intact class and the area under curve value is $=0.99$ ) compared to random forest TPR (Intact class area under the curve 0.89).These differences are statistically different. 
Models prediction power can be judged through likelihood ratio that's why we have summarized the likelihood ratios in Table 7 . Table 7 is showing likelihood ratio.

\section{Discussion and conclusion}

There are many reasons for musculoskeletal disability. After low back pain and neck pain, the third most common cause of musculoskeletal disability is shoulder pain. Frozen shoulder is characterized by pain, stiffness and limited range of motion in shoulder joint. Frozen shoulder condition cannot be identified through X-ray; MRI is carried out to explore this condition. The first description of primary frozen shoulder has been made 150 year ago, but only 16 limited researches has been done to diagnose the underlying cause. According to the cause's symptoms, secondary frozen shoulder problem may occur after trauma or surgery. Little work has been made on the valuable treatment options for frozen shoulder. Due to limited knowledge about the treatment plan in literature, it's really tough for diagnosis of the exact problem and recommendation of best treatment plan. All the treatment plans which are mentioned in a literature have a different success rates. Its estimated value in common population is $2-3 \%$ and a $5-6 \%$ ratio is mentioned by the orthopedics surgeons (Mitchell et al. 2005).

This study also verifies that the frozen shoulder problem mostly occurred in female patients. In this research our anticipated, predictive procedures performed well than the statistical techniques. The specificity and sensitivity ratio of an intelligently predicting frozen shoulder are better in the Naïve Bayes and Logistic Regression models as compared to Random Forest. Our results are similar to recent researches which shows that, mining procedures are more helpful than statistical to accurately diagnose different diseases (Wahbeh et al. 2011; Grossi et al. 2007). However, mining techniques has not been used so much by orthopedic doctors for disease prediction. This limitation enlightens that little researches and studies have been done on a predictive data mining in the field of orthopedic. Numerous orthopedic researches and studies have examined the pathology, treatment and capability of the clinical exam to properly identify frozen shoulder. Previous researches also show that there is extensive value of sensitivity (up to 20-91\%) and specificity (up to $20-100 \%$ ) for disease diagnosing by Arthography method. That is why then arthography results are compared with sonographic signs (Ryu et al. 1993), but no studies has examined the physical examination variable for disease diagnosing. We established that our predictive mining techniques (Naïve Bayesian and the Logistic Regression) are correct for identifying frozen shoulder. These models having specificity is $91-98 \%$, and sensitivity is $96-100 \%$, which is better than earlier researches. If the research shows high sensitivity and moderate specificity, then we can easily use the mining techniques more than statistical for identifying frozen shoulder. We can reduce the unnecessary imaging test ratio and also decrease the false positive rate by data mining procedures.

Table 7 Likelihood ratios

\begin{tabular}{lll}
\hline Model & LR+ & LR- \\
\hline Logistic regression & 11.60 & 0.04 \\
Naïve Bayes & 40 & 0 \\
Random forest & 2.58 & 0.01 \\
\hline
\end{tabular}


All the previous studies related to medical statistics were planned to explore the analysis among group data. If requirement is to do medical diagnosis at a single level, then we cannot use the same procedure due to its limitations (Grossi et al. 2007; Chang 2006). During statistical analysis, researchers do a study on a collection of population to disclose relationships among them. Through evidence-based statistics, we can easily predict a diagnosis at an individual level. Therefore, in our study we used the intelligent data mining model, to give an answer at the specific level of classification (Grossi et al. 2007; Chang 2006; Rygielski et al. 2002).

We have developed an intelligent system by using clinical reports, which can be applied to every individual patient of frozen shoulder. During clinical assessments mostly doctors make diagnosis and recommend treatment on the basis of clinical examination and imaging tests. During physical assessment, doctors frequently face different type of problems. On this basis, it's really difficult for them to determine the disease probability. The classification output of predictive mining was mostly expressed by group method. In this, each individual patient was marked just into one group without knowing that the patients are in the exact class or not. Then, we evaluated the $P$ value against each variable. On the basis of $\mathrm{P}$ value results, we have ignored some irrelevant variable. After $\mathrm{P}$ value calculation, we used significant variables in a model to predict results and also measure model accuracy. To support our predictive results, we calculated the probability of the prediction outcome with LR (Sox and Harold 1996; Dujardin et al. 1994; Sox et al. 1988) and Fagan's theorem (Deeks and Altman 2004; Lang and Secic 1997). The sensitivity and specificity of the Naïve Bayesian model were 100 and $97.5 \%$ (as showed in Table 6) which has given a positive LR (LR+) of 40 and a negative LR (LR-) of 0 . The sensitivity and specificity of the Logistic Regression model were 96.7 and $91.67 \%$ (as showed in Table 6) which has given a positive LR (LR+) of 11 and a negative LR (LR-) of 0.04 (Table 7) (Deeks and Altman 2004; Lang and Secic 1997; Lu et al. 2014).

The positive likelihood result showed that, if a patient visits a hospital with a frozen shoulder there is $11 \%$ time more chance that it has a positive test than those who doesn't. On the other hand, if a patient visits without a diagnosed case of a frozen shoulder, then there is approximately $5 \%$ times more chances that its original test will be negative. Previous studies has recommended that if any model shows its LR+ value greater than ten, there are chances that its original test would be positive. On the other hand, if model results show it LR - value less than 1, there are chances that original test would be negative (Akobeng 2007).

In our study, firstly we calculated the predictive model results and also calculated the likelihood ratios. At the end we have used a Bayesian standard theorem, to evaluate the possibility of presence or absent of a frozen shoulder using a prediction result (intact or no-intact) and a prior probability. On the basis of likelihood ratio, doctors can easily give a rough estimate either a patient has a severe frozen shoulder or not. This will also help to tell the severity of disease before recommending an image test (Espallardo 2003). In Bayesian theorem, the main purpose of likelihood ratio is to change the posterior possibility of having disease, after an outcome is well-known (Gill et al. 2005).

We can easily predict the posterior probability by Fagan nomogram. nomo-gram represents usually through graph, that gives the posterior ratio that a disease is exist or not on the basis of predictive results and prior ratio (Akobeng 2007). Fagan nomo-gram tool 
mark the prior probability on a left side, (a straight line start with a prior ratio of having a disease) and then moved towards the likelihood ratio and at the last intersect at the posterior ration to show ratio of having a disease. For example, if the disease prevalence ratio for a patient is $76 \%$, according to our Logistic Regression model the patient should be in a class of "intact" with an LR+ 11 (Table 7), Firstly, tool marked the prior probability ratio at the left side which is $76 \%$ then draw a straight line (Fig. 3) and marked the point of LR+ $11 \%$ that intersect at the posterior probability of $97 \%$ (Halkin et al. 1998). This result shows that, when the mining model result is "Intact" then the ratio of having a frozen shoulder for this patient increase from 76 to $97 \%$.Conversely, when the model outcome is "no-intact" then the possibility of having the patient an intact decrease from 76 to $11 \%$. Hence, our model outcome help doctors in making diagnostic decision and treatment, mainly if the prior possibility ratio of a frozen shoulder is at the middle. Our model outcomes can be utilized not only to categorize a patient into the "intact" or "nointact" class but also to change the prior possibility in a way to approximate the posterior possibility.

There are also some limitations for which future research is recommended. First, to deal with the problem which can be occurred due to sample size and also more characteristics of patients are required to deal with the generalization issue (Lawrence and Giles 2000). Secondly, more mining models such as SVM (support vector machine) or

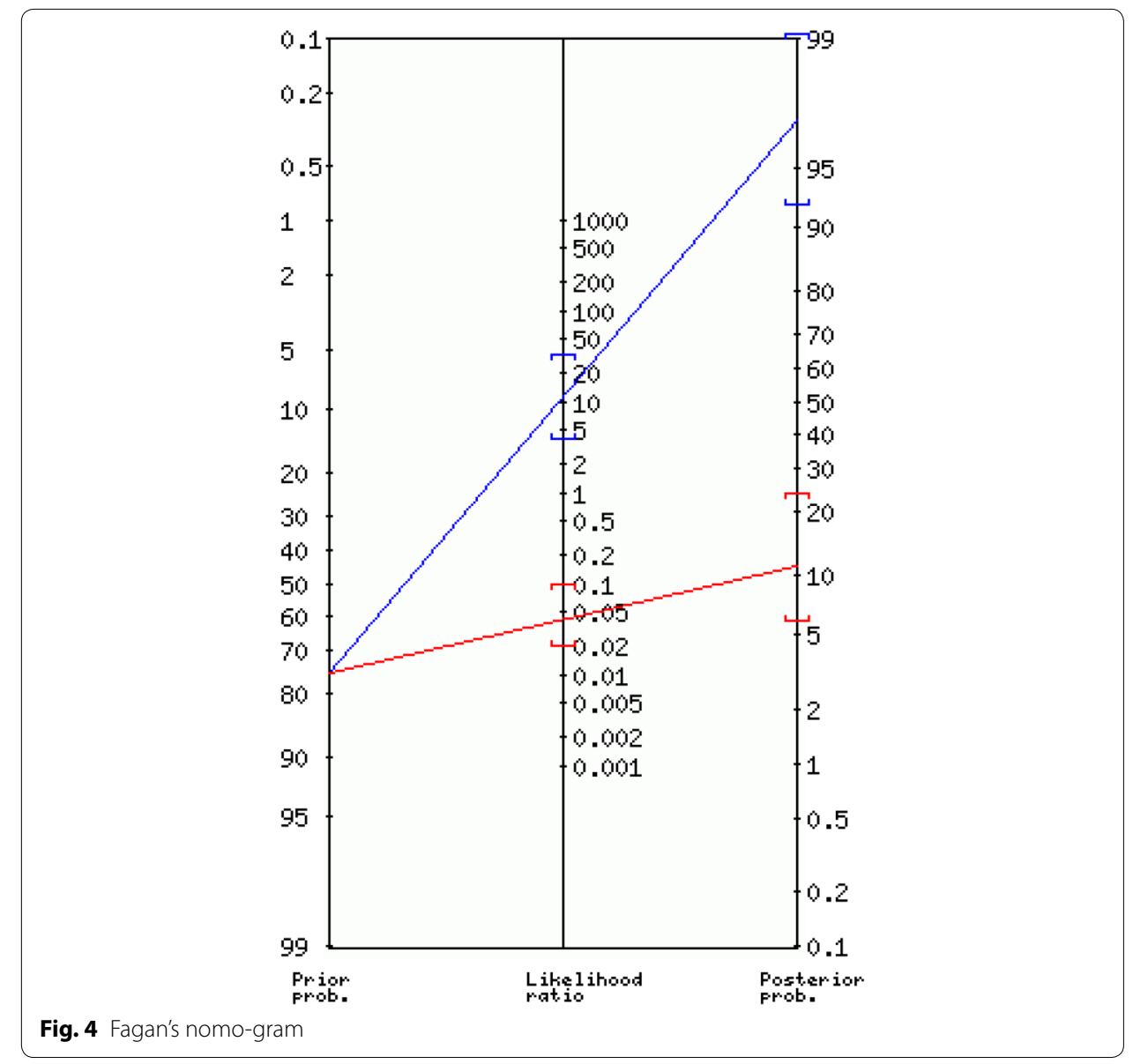


decision tree/neural network should also be used to discover improvement of the prediction. Thirdly, more research is needed to examine whether other medical parameters like Capsular Pattern test or the Lift-off test can be good attributes in affecting the miming model performances. Our research did not focus on a surgery or arthroscopy as main principles, although, the real finding can only be guessed by surgery results. In our study, we used MRI results as a reference standard. Instead of this, the performance and legitimacy of our prediction mining models should be further checked on those patients, who are undergoing for a surgery. Figure 4 shows the Fagan's nomo-gram mapping.

To our information, this study is the initial analysis on an unstructured data with numerous characteristics (age, gender) and disease information (such as disease related, work related), which potentially help the diagnosis of frozen shoulder.

Authors' contributions

$\mathrm{HB}$ and MUA carried out the algorithm designing, conducted experiments, participated in the sequence alignment and drafted the manuscript. WHB and FB participated in the sequence alignment. HB, WHB and MUA participated in the design of the study and performed the statistical analysis. FB validated the algorithms from clinical perspective and provided annotations. All authors read and approved the final manuscript.

\section{Author details}

${ }^{1}$ National University of Sciences and Technology, Islamabad, Pakistan. ${ }^{2}$ Riphah College of Rehabilitation Sciences, Riphah International University Islamabad, Islamabad, Pakistan.

\section{Acknowledgements}

We are thankful to Pakistan Institute of Medical Sciences (PIMS) for their clinical support and help. They also helped us in gathering patient's data.

\section{Competing interests}

The authors declare that they have no competing interests.

Received: 13 June 2016 Accepted: 13 October 2016

Published online: 21 October 2016

\section{References}

Afsar SS, Gulzar M, Idrees M, Ullah Babar I (2014) Results of Intra-articular injection of methylprednisolone in idiopathic adhesive capsulitis. Pak J Surg. 30(1):45-50

Ahmad S, Rafi MS, Siddiqui IA, Hamidi K, Faruq NM (2012) The frequency of adhesive capsulitis in diabetes mellitus patients. Pak J Rehabilit 1(2):49-55

Akobeng AK (2007) Understanding diagnostic tests 2: likelihood ratios, pre-and post-test probabilities and their use in clinical practice. Acta Paediatr 96(4):487-491

Anderson BC, Anderson RJ, Fields KB, Grayzel J (2011) Evaluation of the patient with shoulder complaints. UpToDate, Waltham

Bagley SC, White H, Golomb BA (2001) Logistic regression in the medical literature: standards for use and reporting, with particular attention to one medical domain. J Clin Epidemiol 54(10):979-985

Bellazzi R, Zupan B (2008) Predictive data mining in clinical medicine: current issues and guidelines. Int J Med Inform 77:81-97

Bengio Y, Grandvalet Y (2004) No unbiased estimator of the variance of k-fold cross-validation. J Machine Learn Res 5:1089-1105

Binder Al, Bulgen DY, Hazleman BL, Roberts S (1984) Frozen shoulder: a long-term prospective study. Ann Rheum Dis 43(3):361-364

Breiman L, Friedman J, Stone CJ, Olshen RA (1984) Classification and regression trees. CRC Press, Boca Raton

Bulgen DY, Binder Al, Hazleman BL, Dutton J, Roberts S (1984) Frozen shoulder: prospective clinical study with an evaluation of three treatment regimens. Ann Rheum Dis 43(3):353-360

Chang L (2006) Applying data mining to predict college admissions yield: a case study. New Direct Institut Res 2006(131):53-68

Clarke GR, Willis LA, Fish WW, Nichols PJR (1974) Assessment of movement at the glenohumeral joint. Orthopaedics 7(2):55-71

Crubbs N (1993) Frozen shoulder syndrome: a review of literature. J Orthop Sports Phys Ther 18(3):479-487

Deeks JJ, Altman DG (2004) Diagnostic tests 4: likelihood ratios. BMJ 329(7458):168-169

Değerlendirmesi OBR (2014) Radiological assessment of the shoulder region

Delen D, Walker G, Kadam A (2005) Predicting breast cancer survivability: a comparison of three data mining methods. Artif Intell Med 34(2):113-127

Dias R, Cutts S, Massoud S (2005) Frozen shoulder. Br Med J 331(7530):1453 
Dujardin B, Van den Ende J, Van Gompel A, Unger JP, Van der Stuyft P (1994) Likelihood ratios: a real improvement for clinical decision making? Eur J Epidemiol 10(1):29-36

Espallardo N (2003) Decisions on diagnosis in family practice: use of sensitivity, specificity, predictive values and likelihood ratios. Asia Pac Family Med 2(4):229-232

Gill CJ, Sabin L, Schmid CH (2005) Why clinicians are natural bayesians. Br Med J Int 330(7499):1080-1083

Griffith J (2000) Artificial neural networks: are they ready for use as clinical decision aids? Med Decis Making 20(2):243-244

Grossi E, Mancini A, Buscema M (2007) International experience on the use of artificial neural networks in gastroenterology. Digest Liver Dis 39(3):278-285

Halkin A, Reichman J, Schwaber M, Paltiel O, Brezis M (1998) Likelihood ratios: getting diagnostic testing into perspective. QJM 91(4):247-258

Han J, Pei J, Kamber M (2011) Data mining: concepts and techniques. Elsevier, Amsterdam

Kassirer JP (1989) Our stubborn quest for diagnostic certainty. N Engl J Med 320(22):1489-1491

Kohavi R (1995) A study of cross-validation and bootstrap for accuracy estimation and model selection. Int Joint Conference Artif Intell. 14(2):1137-1145

Lang TA, Secic M (1997) How to report statistics in medicine. American College of. Physicians, Philadelphia

Lawrence S, Giles CL (2000) Overfitting and neural networks: conjugate gradient and backpropagation. IEEE International Joint Conference on Neural networks, Proceedings of the IEEE-INNS-ENNS, vol 1. pp 114-119)

Lin CC, Ou YK, Chen SH, Liu YC, Lin J (2010) Comparison of artificial neural network and logistic regression models for predicting mortality in elderly patients with hip fracture. Injury 41(8):869-873

Lin CS, Chang CC, Chiu JS, Lee YW, Lin JA, Mok MS, Li YC (2011) Application of an artificial neural network to predict postinduction hypotension during general anesthesia. Med Decis Making 31(2):308-314

Lu HY, Huang CY, Su CT, Lin CC (2014) Predicting rotator cuff tears using data mining and bayesian likelihood ratios. PLoS ONE 9(4):e94917

Manske RC, Prohaska D (2008) Diagnosis and management of adhesive capsulitis. Curr Rev Musculoskeletal Med 1(3-4):180-189

Manske RC, Prohaska D (2010) Clinical commentary and literature review: diagnosis, conservative and surgical management of adhesive capsulitis. Shoulder Elbow 2(4):238-254

Mao CY, Jaw WC, Cheng HC (1997) Frozen shoulder: correlation between the response to physical therapy and follow-up shoulder arthrography. Arch Phys Med Rehabil 78(8):857-859

Mitchell C, Adebajo A, Hay E, Carr A (2005) Shoulder pain: diagnosis and management in primary care. Br Med J 331(7525):1124-1128

Murdoch TB, Detsky AS (2013a) The inevitable application of big data to health care. JAMA 309(13):1351-1352

Murdoch TB, Detsky AS (2013b) The inevitable application of big data to health care. JAMA 309(13):1351-1352

Neviaser JS (1945) Adhesive capsulitis of the shoulder. J Bone Joint Surg Am 27(2):211-222

Oh CH, Schweitzer ME, Spettell CM (1999) Internal derangements of the shoulder: decision tree and cost-effectiveness analysis of conventional arthrography, conventional MRI, and MR arthrography. Skeletal Radiol 28(12):670-678

Pauker SG, Kassirer JP (1980) The threshold approach to clinical decision making. N Engl J Med 302(20):1109-1117

Ramesh AN, Kambhampati C, Monson JRT, Drew PJ (2004) Artificial intelligence in medicine. Ann R Coll Surg Engl 86(5):334

Rygielski C, Wang JC, Yen DC (2002) Data mining techniques for customer relationship management. Technol SoC 24(4):483-502

Ryu KN, Lee SW, Rhee YG, Lim JH (1993) Adhesive capsulitis of the shoulder joint: usefulness of dynamic sonography. J Ultrasound Med 12(8):445-449

Shaffer B, Tibone JE, Kerlan RK (1992) Frozen shoulder. A long-term follow-up. J Bone Joint Surg Am 74(5):738-746

Sharma RK, Bajekal RA, Bhan S (1993) Frozen shoulder syndrome. Int Orthop 17(5):275-278

Sox MD, Harold C (1996) The evaluation of diagnostic tests: principles, problems, and new developments. Annu Rev Med 47(1):463-471

Sox HC, Blatt MA, Higgins MC, Marton KI (1988). Measuring the outcome of care. Med Decis Mak 201-237

Wahbeh AH, Al-Radaideh QA, Al-Kabi MN, Al-Shawakfa EM (2011) A comparison study between data mining tools over some classification methods. International Journal of Advanced Computer Science and Applications 8(2):18-26

Weinstein MC, Fineberg HV, Elstein AS, Frazier HS, Neuhauser D, Neutra RR, McNeil BJ (1980) Clinical Decision Analysis. WB Saunders Co., Philadelphia

Witten IH, Frank E (2005) Data mining: practical machine learning tools and techniques. Morgan Kaufmann, Burlington Woodward TW, Best TM (2000) The painful shoulder: part I clinical evaluation. Am Fam Physician 61(10):3079-3089 